\title{
A CHARACTERIZATION OF CONVEX SURFACES WHICH ARE $L$-SETS ${ }^{1}$
}

\author{
E. O. BUCHMAN AND F. A. VALENTINE
}

\begin{abstract}
If the surface of a bounded three dimensional convex body has the property that each pair of its points can see some third point via the surface, then with a single exception the body must be a finite cone with a convex base. The exceptional shape is that of a solid hexahedron with six triangular plane faces formed as the union of two tetrahedra having a congruent face in common.
\end{abstract}

A set $S$ in a linear space is an $L$-set if each pair of its points can see some point of $S$ via $S$. In other words, if $x \in S, y \notin S$, there exists a point $z \in S$ (which may vary with $x$ and $y$ ) such that $x z \subset S, y z \subset S$. Although much is known about $L$-sets in the plane $E^{2}$ ([1], [2], [3], [4], [5], [6]), relatively little is known about $L$-sets in Euclidean $n$-space $E^{n}$ when $n \geqq 3$. However, here we have succeeded in characterizing $L$-surfaces (surfaces which are $L$-sets) which are boundaries of compact convex bodies in $E^{3}$.

In order to observe intuitively the simple geometric nature of this situation, duality and elementary graph theory reveal that the following concepts are useful.

Definition 1. A face of a convex body $B$ is the intersection of $B$ with one of its planes of support. An exposed point $x$ of $B$ is a point which is also a face of $B$. (Hence, there exists a plane of support $H$ of $B$ such that $H \cap B=x$.) Let $\exp B$ be the set of exposed points of $B$.

REMARK. In the following treatment we prefer to use "exposed points" instead of "extreme points" since an "exposed point" is always a minimal face whereas an "extreme point" $e$ of $B$ (i.e., $B \sim e$ is convex) is not necessarily even a face, let alone minimal. A minimal face is, of course, a face which does not contain a smaller face, and a corresponding definition holds for "maximal face". The following elementary theorem shows the graph theoretic nature of $L$-surfaces.

THeOREM 1. Suppose $S$ is the surface of a compact convex set $B$ with nonempty interior in Euclidean $n$-space $E^{n}$.

Received by the editors July 16, 1971 and, in revised form, September 13, 1972.

AMS (MOS) subject classifications (1970). Primary 52A15, 52A25.

Key words and phrases. Convex surface, $L$-set, face, exposed point, convex cone.

${ }^{1}$ The preparation of this paper was supported in part by NSF Grant GP-22518.

(c) American Mathematical Society 1973 
Then $S$ is an L-set if and only if every two maximal faces of $S$ have nonempty intersection.

Proof. To prove this we use the following.

Notation. The interior, closure, boundary and convex hull of a set $K \subset E^{n}$ are denoted by int $K, \mathrm{cl} K$, bd $K$ and conv $K$ respectively. The empty set is denoted by $\varnothing$, and $x y$ is the closed segment joining $x \in E^{n}$ to $y \in E^{n}$.

(Necessity). Suppose $F_{i}(i=1,2)$ are two maximal faces of $B$. Since $S$ is an $L$-set, $F_{i}$ contains at least one segment. Let $H_{i}$ be the minimal flat containing $F_{i}$. Choose points $x_{i} \in$ real int $F_{i}$ where "rel int" is the interior relative to $H_{i}$. Since $S=$ bd $B$ is an $L$-set, there exists a point $z$ such that $x_{i} z \subset S(i=1,2)$. We can prove that $x_{i} z \subset F_{i}$.

Since $F_{i}$ is convex and since $x_{i} \in$ rel int $F_{i}$, we can express $F_{i}$ as the union of all those line segments $x y$ lying in $F_{i}$ and having $x_{i}$ in their relative interiors. However, if $x_{i} \in$ rel int $x y, x \in F_{i}, y \in F_{i}$ then $x_{i} z \subset S, x y \subset S$, and the convexity of $B$ imply that $\operatorname{conv}(x \cup y \cup z) \subset S$. Consequently $\operatorname{conv}\left(F_{i} \cup z\right) \subset S$, which, in turn, implies $z \in F_{i}(i=1,2)$, since $F_{i}$ is a maximal face of $B$. Hence, we have $F_{1} \cap F_{2} \neq \varnothing$.

The sufficiency is immediate.

To complete the theory we need the following concept.

DEFINITION 2. If $F$ is a two-dimensional compact convex set and if $p$ is a point not in the plane containing $F$, then $\operatorname{conv}(p \cup F)$ is called a solid finite cone.

Our main theorem is the following.

THEOREM 2. Suppose $B$ is a bounded closed convex set in $E^{3}$ having nonempty interior.

Also suppose that either (a) "Every two maximal faces of $B$ intersect", or dually, (b) "Every two exposed points of B lie on a common face of B".

Then the following holds. Either

(1) $B$ is a solid finite cone, or

(2) if (a) holds then $B$ is a solid hexahedron with six triangular plane faces; if (b) holds, then $B$ is a solid pentahedron with six vertices each of valence three (each vertex is incident with exactly three edges).

Observe that a finite cone is self-dual whereas the hexahedron and the pentahedron in (2) are dual polyhedra. The converse is immediate.

Proof of Theorem 2. Because of polar duality, it is sufficient to derive, in order, the following consequences of hypothesis (b). I. Of every five exposed points of $B$ at least four are coplanar. II. Either (i) all but one of the exposed points of $B$ are coplanar or (ii) $B$ has exactly six exposed points. III. Theorem 2 follows. 
Proof of I. Assume that five exposed points $x, y_{i}(i=1,2,3,4)$ of $B$ exist, no four of which are coplanar. Since no three of the edges $x y_{i}$ $(i=1, \cdots, 4)$ are then coplanar, and since the four points $y_{i}(i=1, \cdots, 4)$ are not coplanar, it is easy to verify that one of the six edges $y_{i} y_{j}(i, j=$ $i, \cdots, 4, i \neq j)$ is an interior diagonal of $B$. This violates hypothesis (b), and $I$ has been proved. This is illustrated in Figure 1.

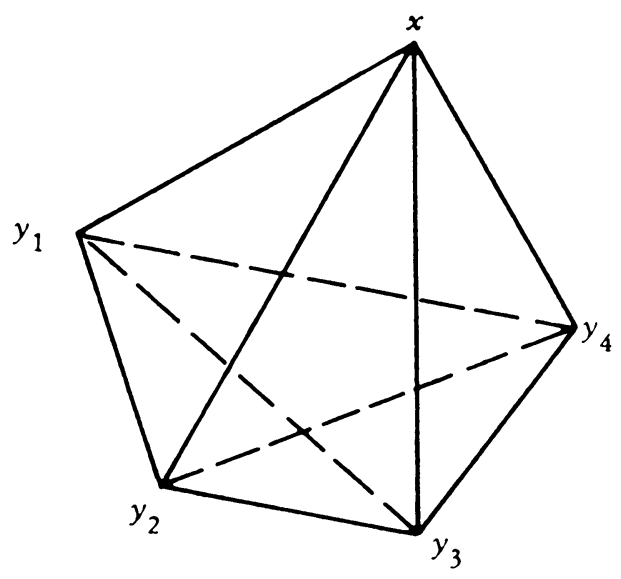

FIGURE 1

Proof OF II. If the set of exposed points $\exp B$ has only four points, then II follows trivially. Hence, suppose $\exp B$ contains at least five noncoplanar points. Result I implies that some four of these, say $x_{1}, x_{2}, x_{3}, x_{4}$, are coplanar, where we assume a cyclic ordering in the plane $P$ containing them. Also let $x$ be a point of $\exp B$ not in $P$. If all of the remaining points of $\exp B$ lie in $P$, then (i) of II follows. Hence, suppose there exists a point $y \in \exp B$ with $y \notin P, y \neq x$. If $x y$ is coplanar with $x_{1} x_{3}$ then the diagonals of the quadrilateral $x y x_{1} x_{3}$ are interior diagonals of $B$, and this violates hypothesis (b). With a symmetric argument this implies that $x y$ is not coplanar with either $x_{1} x_{3}$ or $x_{2} x_{4}$. This and result I imply that $x y$ is coplanar separately with two opposite edges of the quadrilateral $x_{1} x_{2} x_{3} x_{4}$. If $\exp B$ contains a seventh point $z$, we may if necessary relabel the points $x, y, x_{1}$, $x_{2}, x_{3}, x_{4}$ to insure that $z \notin P$, and then the preceding sentence applied to $x y, y z$, and $x z$, respectively, implies that each of them is coplanar with each of two opposite edges of the quadrilateral $x_{1} x_{2} x_{3} x_{4}$. This is only possible if $x, y, z$ are all collinear, which violates the fact that $x, y, z$ are all exposed points of $B$. Hence, $z$ cannot exist, so that $\exp B=\left\{x, y, x_{1}, x_{2}, x_{3}, x_{4}\right\}$.

Proof OF III. If all but one point of $\exp B$ lie in a plane, then $B$ is a solid cone, being the closed convex hull of $\exp B$ [7]; otherwise, result II 
shows that $B$ has exactly six exposed points contained in three lines which are either parallel or concurrent. In the latter case, recalling that $B$ has no internal diagonals, it follows readily that the closed convex hull of $\exp B$ does not contain the point of concurrence (if any) of these three lines, and, hence, $B$ is a solid pentahedron with six vertices each of valence three (see Figure 2). Theorem 2 follows because of duality.
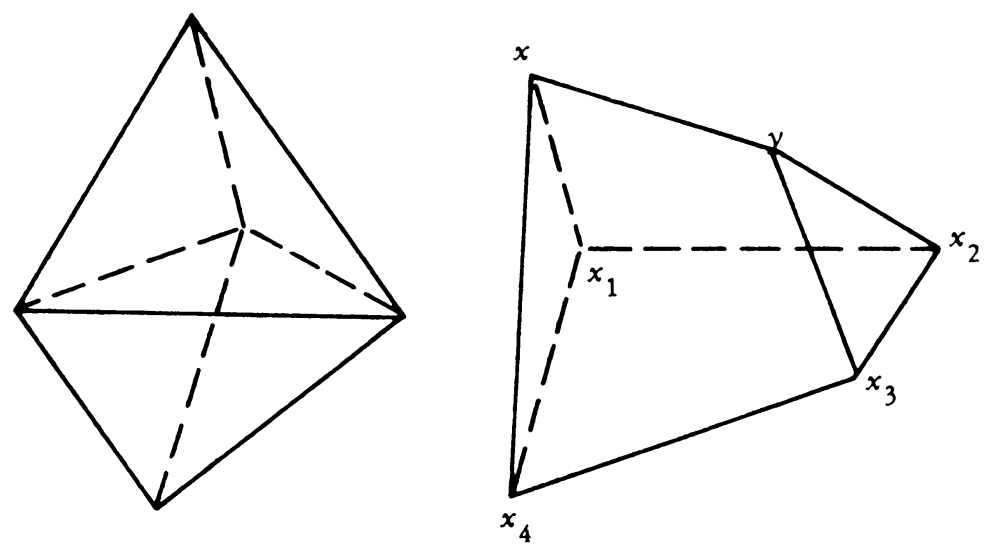

FIGURE 2. Dual solids

REMARK. Since the hypothesis (a) is essentially combinatorial, it is clear that any nonconvex polyhedron with convex faces whose incidence relationships coincide exactly with those of a corresponding convex polyhedron $B$ will share with $B$ the property of having surfaces which are $L$-sets or not.

It is of interest to observe that our theorem shows that the boundary $S$ of $B$ cannot be expressed as the union of line segments every two of which have a nonempty intersection. Mackie [3] has shown that a simply connected compact $L$-set in the plane $E^{2}$ can be expressed as the union of line segments every two of which have nonempty intersection. However, Theorem 1 does show that the surface $S$ is an $L$-set if and only if it can be expressed as the union of convex subsets every two of which have nonempty intersection, even though $S$ is not simply connected relative to $E^{3}$ [2].

\section{BIBLIOGRAPHY}

1. A. M. Bruckner and J. B. Bruckner, On $L_{n}$ sets, the Hausdorff metric and connectedness, Proc. Amer. Math. Soc. 13 (1962), 765-767. MR 25 \#5452.

2. A. Horn and F. A. Valentine, Some properties of L-sets in the plane, Duke Math J. 16 (1949), 131-140. MR 10, 468. 
3. D. G. Mackie, The structure of L-sets, Dissertation, University of California, Los Angeles, Calif., 1965.

4. A. G. Sparks, Characterizations of the generalized convex kernel, Proc. Amer. Math. Soc. 27 (1971), 563-565. MR 43 \#5413.

5. N. M. Stavrakis, W. R. Hare and J. W. Kenelly, Two cells with $n$ points of local nonconvexity, Proc. Amer. Math. Soc. 27 (1971), 331-336. MR 42 \#5163.

6. F. A. Valentine, Local convexity and $L_{n}$ sets, Proc. Amer. Math Soc. 16 (1965), 1305-1310. MR 32 \#2976.

7. - Convex sets, McGraw-Hill Series in Higher Math., McGraw-Hill, New York, 1964. MR 30 \#503.

Department of Mathematics, California State College, Fullerton, CaliFORNIA 92631

Department of Mathematics, University of California, los Angeles, CaliFORNIA 90024 